\title{
Jean-Pierre DUPUY, La Marque du sacré
}

Paris, Éd. Carnets Nord, coll. Essai, 2008, 281 p.

\section{Stefan Goltzberg}

\section{(2) OpenEdition \\ 1 Journals}

Édition électronique

URL : http://journals.openedition.org/questionsdecommunication/314

ISSN : 2259-8901

\section{Éditeur}

Presses universitaires de Lorraine

\section{Édition imprimée}

Date de publication : 1 décembre 2010

Pagination : 297-298

ISBN : 978-2-8143-0056-9

ISSN : 1633-5961

\section{Référence électronique}

Stefan Goltzberg, « Jean-Pierre DuPuY, La Marque du sacré », Questions de communication [En ligne],

18 | 2010, mis en ligne le 09 mai 2012, consulté le 30 août 2019. URL : http://journals.openedition.org/ questionsdecommunication/314 
questions de communication, 2010, 18

\section{Jean-Pierre Dupur, La Marque du sacré.}

Paris, Éd. Carnets Nord, coll. Essai, 2008, 28I p.

Dans cet ouvrage, l'auteur livre à la fois un « carnet de bord » et un « polar métaphysique et théologique » où il traque la marque du sacré dans de nombreux domaines qui ont pour points communs de vouloir s'en affranchir : l'économie, l'éthique, la psychologie, la philosophie politique, la biologie. La question fondamentale est celle des figures du sacré dans un monde où, précisément, la transcendance fait prétendument défaut. C'est que, loin d'évacuer le sacré, les systèmes en question sont d'autant plus travaillés par un sacré qui ne dit pas toujours son nom, mais qui réapparaît sous le nom, par exemple, de hasard. En dialogue avec l'œuvre de René Girard, ce livre explore le sacré comme mécanisme d'auto-extériorisation de la violence humaine. L'auteur dénonce « l'impuissance des rationalismes contemporains à appréhender la forme de l'autotranscendance » (p. 27), impuissance ayant pour cause le déni d'un sacré qui les constitue. La désacralisation du monde laisse démunis face à la violence : le sacré n'est pas la source de la violence mais le dispositif qui la contient - ce verbe doit être entendu au deux sens de englobe et repousse. L'apocalypse occupe une place de choix dans l'économie du livre. L'auteur ayant souscrit dans un ouvrage précédent (Pour un catastrophisme éclairé, Paris, Éd. Le Seuil, 2002) à un catastrophisme éclairé, il revient à présent sur ce qui n'est pas tant un événement qu'une structure: la catastrophe est véritablement une vision du monde. Qu'on se comprenne, la catastrophe doit être présente à l'esprit afin qu'elle ne se réalise pas; il s'agit d'une sorte de prophétie auto-déréalisatrice, d'une prédiction qui est vouée, si elle est prise au sérieux, à ne pas se réaliser. Précision : à se réaliser le plus tard possible. En effet, « dans la tradition apocalyptique vulgaire, l'apocalypse est la fin des temps ». Pourtant, Jésus lui-même use du langage apocalyptique, non pour valider la lecture historique, mais pour « désacraliser l'apocalypse ». L'auteur invite à subvertir de l'intérieur également la crise actuelle: c'est cela qu'il appelle le « catastrophisme éclairé » (pp. 44-45). Jean-Pierre Dupuy est donc tout sauf un Nostradamus; il met simplement en garde contre ce que l'apocalypse révèle : la violence des hommes, pas uniquement celle de la nature, de la barbarie ou de Dieu, mais des hommes eux-mêmes. D'une manière générale, l'auteur invite le lecteur à dire autrement la crise et le monde contemporain. Un exemple éloquent de ceci suffit: « II est trompeur de parler de crise énergétique : nous avons trop de ressources fossiles » (p. 5l). L'auteur ne nie pas qu'il $y$ ait une crise mais remet en question les grilles de lecture communément simplistes ou manichéennes, auxquelles échappe la structure du sacré et de la violence.: « Nous avons plus à craindre aujourd'hui les industriels du bien que les méchants » (p. 53).

En caractérisant la « science » comme une « théologie qui s'ignore» (chapitre 2), l'auteur n'adopte pas une posture de condamnation de la science, mais insiste sur la nécessité de repenser le concept 
d'autonomie. Une société totalement autonome, selon les termes du problème théologico-politique, devrait faire l'économie de toute transcendance; or, toute société autonome se limite elle-même. Nous sommes donc dans « l'incapacité de penser l'autonomie dans sa radicalité maximale » (p. 97). Dans ce monde visant à l'autonomie, ce monde sans Dieu, se posent de nombreux problèmes bioéthiques. Le transhumanisme, qui pousse l'espèce humaine à se dépasser grâce à la technique, serait « typiquement l'idéologie d'un monde sans Dieu ». Et d'ajouter que cette doctrine transhumaniste dualiste - se trouve en « parfaite contradiction » avec la métaphysique moniste qui sous-tend les sciences cognitives (p. 108). Le chapitre 3 prend pour thème « La religion, nature ou surnature? » Se définissant volontiers comme « chrétien intellectuel», l'auteur voit dans l'Évangile « la condition de possibilité de toute science humaine ». Le christianisme serait d'ailleurs « la religion de la fin des religions », ce qui lui donne l'occasion d'élever une critique contre une des disciplines sur lesquelles il a déà écrit, le cognitivisme (pp. 122-123). Celui-ci, assimilant le christianisme à une religion parmi d'autres, passe à côté de la question. Les sciences cognitives seraient de surcroît victimes d'une notion présentée par l'auteur comme privée de sens : la pertinence (p. 135). Plutôt que des concepts cognitivistes, il serait préférable de s'inspirer des notions articulées par René Girard, notamment celle de sacrifice. Et de rappeler un paradoxe bien connu des anthropologues: il est criminel de tuer la victime parce qu'elle est sacrée, mais la victime ne serait pas sacrée si on ne la tuait pas. On le voit aisément, la pensée sacrificielle mène au «brouillage des catégories »: la description anthropologique enjoint de confondre précisément ce que l'analyse distingue (pp. |39-|4|). Le chapitre 4 traque dans le hasard la marque du sacré. Ce hasard qui, pour les Modernes, est assimilé à l'incertitude, préside dans les sociétés non modernes, aux institutions de base de la société. Ce phénomène apparait dans le choix du bouc émissaire, le partage des terres, le choix du roi du carnaval ou encore la méthode démocratique chez les Anciens. Loin de s'identifier au hasard moderne, dépourvu de sens, le hasard primitif prend une part active dans le processus de décision : il est chargé d'intentionnalité. lci, la question est posée au sujet d'un couple souhaitant adopter sans choisir l'enfant et sans tirer au sort. L'auteur démontre que l'on peut faire appel au hasard, dans l'exacte mesure où les conditions produisent une extériorité et une transcendance (p. 196). Nouvelle marque du sacré, le hasard suscite le paradoxe du vote, variante du paradoxe du sorite (ou du tas) : sauf cas très improbable, le vote d'un unique individu ne changera jamais rien au résultat. Dès lors, pourquoi voter? Le déplacement ayant un coût, le citoyen ne devrait pas voter! L'auteur critique le mépris avec lequel certains psychologues ont qualifié de magiques les motivations des électeurs: si je vote, d'autres iront voter, et voteront sans doute comme moi. Pour l'auteur de la Marque du sacré, l'épistémologie n'est pas simpliste, ni même simple, mais complexe. Ainsi est-il l'un des rares auteurs à échapper aux explications d'école, qu'il s'agisse des individualistes ou des holistes. II s'est déclaré pour un individualisme méthodologique complexe, complexité qu'il ne perd pas de vue dans les chapitres dont il n'a pas été question ici : sur les théories de la justice, la menace nucléaire ou encore les problèmes d'autoréférence dans le film Vertigo (Hitchcock, 1958).

En essayant de retisser tous les fils de ce très bel ouvrage - la loterie, le sacrifice, l'épistémologie, la psychologie cognitive, la théologie, la bombe atomique, le cinéma, et beaucoup d'autres - le lecteur comprendra à quel point il s'agit d'une enquête, d'un « polar métaphysique et théologique ». II est très rare de trouver sous la plume d'un seul auteur, cette capacité de croiser les disciplines. Si le mot transdisciplinaire mérite d'être employé au sens fort, c'est au sujet des pages de La marque du sacré.

Stefan Goltzberg

Université libre de Bruxelles stefgoltz@gmail.com 